\title{
Correlates and short-term prognostic value of plasma n-terminal pro-brain natriuretic peptide levels among heart failure patients: A prospective cohort study
}

Mark Mayala ( $\square$ markmayala90@gmail.com )

Muhimbili University of Health and Alied Sciences

Khuzeima Khanbhai

Jakaya Kikwete Cardiac Institute

Ponsian Peter

Muhimbili University of Health and Alied Sciences

Pilly Chillo

Muhimbili University of Health and Alied Sciences

\section{Research Article}

Keywords: Natriuretic peptides, N-terminal pro-brain natriuretic peptide, New York heart association functional status, Receiver operating characteristic curve

Posted Date: November 1st, 2021

DOI: https://doi.org/10.21203/rs.3.rs-1036087/v1

License: (9) This work is licensed under a Creative Commons Attribution 4.0 International License.

Read Full License 


\section{Abstract}

\section{Background}

Studies conducted in developed countries have found measurements of plasma N-terminal pro-brain natriuretic peptide (NT-proBNP) to be important in evaluating heart failure (HF) prognosis, however this has not yet been studied in sub-Saharan Africa. The aim of this study was to determine clinical correlates and short-term prognostic value of plasma NT-proBNP levels among HF patients.

\section{Methodology}

This was a hospital-based prospective cohort study conducted at Jakaya Kikwete Cardiac Institute, Tanzania from June to December 2020. Patients were consecutively enrolled when they fulfilled the inclusion criteria. Clinical details and NT-proBNP levels were measured at baseline and at 30-day followup. Pearson's chi square test was used to associate New York Heart Association (NYHA) functional class and NT-proBNP levels, while Spearman's correlation coefficient was used to correlate between left ventricular ejection fraction (LVEF) and NT-proBNPlevels. Receiver Operating Characteristic (ROC) curves were drawn to determine the best prognostic cut off points of NT-proBNP levels for the different clinical outcomes. A P-value of $<0.05$ was considered statically significant.

Results

$155 \mathrm{HF}$ patients were enrolled. Their mean \pm SD age was $48 \pm 16$ years, $52.3 \%$ were male and their mean \pm SD LVEF was $37.3 \pm 10.7 \%$. At baseline, the median (IQR) NT-proBNP levels was $7654 \mathrm{pg} / \mathrm{ml}(2289$, $16000)$, and the levels dropped to $3383 \mathrm{pg} / \mathrm{ml}(731,9785)$ after 1 month. Baseline plasma levels of $N T$ proBNP increased as the NYHA functional class worsened, $(P=0.018)$, and with decreasing LVEF $(r=$ $-0.65, \mathrm{p}<0.05$ ). The ROC curve identified an overall cut-off point for poor prognosis at $18000 \mathrm{pg} / \mathrm{ml}$ with $54.4 \%$ sensitivity and $93.7 \%$ specificity (area under the curve (AUC): 0.8 ). The NT-proBNP cut-off point for mortality was $24500 \mathrm{pg} / \mathrm{ml}$ with $100 \%$ sensitivity and specificity of $92.54 \%$ (AUC: 0.958 ). The ROC analysis also identified levels of NT-proBNP of $\geq 7899 \mathrm{pg} / \mathrm{ml}$ to predict re-hospitalization, with $76 \%$ sensitivity and $60 \%$ specificity (AUC: 0.68 ), while levels $\geq 18762.1 \mathrm{pg} / \mathrm{ml}$ predicted long hospital stay with a sensitivity of $100 \%$ and specificity of $85.62 \%$ (AUC: 0.939 ).

\section{Conclusion}

These results demonstrate that NT-proBNP is a powerful measure to predict readmission, mortality and long hospital stay in HF patients and can facilitate discussions with patient prognosis, decisions regarding interventions, and continuity of care.

\section{Background}

Heart failure (HF) has currently been a major and growing public health problem, affecting almost 26 million people worldwide and there is a pressing need to improve outcomes and decrease costs. Within 
the past few years, a large amount of epidemiological data has indicated that HF is a common, underdiagnosed, and serious condition with a dismal prognosis, comparable with several forms of cancer.'

The INTER-CHF Study in 2016, done in sub-Saharan Africa (SSA), estimated the prevalence of HF to be $5 \%$ and increasing. This can be explained by a shift over the past several years in the cardiovascular disease profile that has been observed. This was reflected by a recent largest multicentre registry for HF in Africa. The sub-Saharan Africa Survey of Heart Failure (THESUS-HF) study, which characterized the causes, treatment and short-term outcomes in 1006 Africans from nine SSA countries. The data from this study, highlighted hypertension as a rising cause of HF (from $23 \%$ to $43 \%$ ), followed by cardiomyopathies (from $20 \%$ to $29 \%$ ), rheumatic heart disease (from $22 \%$ to $17 \%$ ), and ischemic heart disease (from $2 \%$ to $8 \%$ ).

In an attempt to facilitate and improve HF diagnosis, of which is clinical-based (Framingham criteria) and by echocardiographic evaluation of left ventricular (LV) systolic function, biochemical substances have been suggested as alternative markers. Efforts have focused on the natriuretic peptides (NPs), among which are brain natriuretic peptide (BNP) and its amino-terminal portion $\mathrm{N}$-terminal pro-brain natriuretic peptide (NT-proBNP). ${ }^{-}$Since HF is a chronic progressive condition, these biomarkers have as well been used to assess its prognosis. Majority of the studies that have been done, have used BNP as the biomarker though comparing techniques have shown that there is a good equivalence between both BNP and NT-proBNP methods, although NT-proBNP values in $\mathrm{pg} / \mathrm{ml}$ are approximately 8 -fold higher than those of BNP.

Cheng and colleagues demonstrated that patients with a significant decrease of BNP at discharge compared with admission BNP levels had better outcomes, whereas BNP levels dropped minimally during hospitalization in patients that were re-hospitalized within 30 days. Besides, Cohen Solal and colleagues demonstrated that patients with a decrease of $30 \%$ or higher BNP value from baseline, at follow-up had reduced mortality risk compared with those with little or no BNP decrease.

Although plasma NT-proBNP can be used to predict further cardiovascular events, re-hospitalization and mortality in HF patients, in Tanzania, natriuretic peptides have not been widely used, not only as a diagnostic biomarker but neither as a prognostic biomarker. In response to this problem, our study proposes to bring awareness to the medical personnel on the importance of natriuretic peptides as a prognostic tool for HF patients. We therefore examined the association between plasma NT- pro BNP levels and NYHA functional status, and its correlation with left ventricular ejection fraction, among other variables in a cohort of Tanzanian patients with HF. Furthermore, the ability of NT-proBNP to predict clinical outcomes at admission and at 1-month post discharge was assessed.

\section{Methodology}


This was a hospital-based prospective cohort study, done at Jakaya Kikwete Cardiac Institution (JKCl), Dar-es-Salaam, Tanzania. JKCl is one of a national tertiary level referral hospital basing mainly on cardiac diseases, both medical and surgical. Patients were consecutively sampled until a sample size of 155 patients was met, that included all patients diagnosed with heart failure aged 18 years and above by the attending physician and excluded patients with chronic kidney disease. It was conducted from June to December 2020. This study aimed to determine the clinical correlates and short-term prognostic value of plasma NT- proBNP levels among HF patients and specifically to associate between plasma NTproBNP levels and NYHA functional status and also to correlate the plasma NT- proBNP levels and left ventricular ejection fraction. The primary outcome was the overall clinical progression, re-hospitalization and mortality at 1 month follow up. Ethical clearance to conduct the study was obtained from Muhimbili University of Health and Allied Sciences' Ethical Review Board. Permission to do the study was obtained from $\mathrm{JKCl}$ management. Informed consent was obtained from all study participants before they were enrolled in the study. Cases eligible to participate in the study were included only after being provided with informed consent.

\section{Data Collection}

Two different strategies for assessing NT-proBNP were examined: Admission levels and the percent change in NT-proBNP levels from admission to follow up. For all patients, a blood sample was taken within 12 hours of admission and this was considered as admission NT-proBNP. Follow up NT-proBNP was done at 1 month after the baseline test was taken. Other baseline laboratory results like haemoglobin level, creatinine level as well as blood urea nitrogen (BUN) were obtained from patients' files and recorded in data collection forms. Echocardiogram results were obtained from detailed echocardiogram examination done within the index admission.

\section{Analysis}

Data was entered on IBM SPSS version 26. Descriptive statistics were analysed using frequency for categorical variables and median (IQR) and mean \pm SD for numerical variables. Chi square test was used to assess between NYHA Class and NT-proBNP, and p-value of less than 0.05 was considered statistically significant. Spearman ranking correlation coefficient was used to assess relationship between LVEF and other continuous variables with NT-proBNP. Receiver Operating Characteristic's (ROC) curve was used to determine the best cut-off points for the prognostic ability of plasma NT-proBNP for readmission, mortality, poor outcome and long hospital stay

\section{Results}

A total of 180 patients with HF were screened for eligibility during the study period, of whom 155 (86.1\%) fulfilled the inclusion criteria and were enrolled. Patient baseline demographic and clinical characteristics are reported in table 1 . The mean age of the study patients was $48 \pm 16 y e a r s$ (range 18-81) years, and 81 (52.3\%) of the patients were males. Most study patients were living in Dar es Salaam 108 (69.7\%), were married $112(72.3 \%)$ and were college graduates 71 (45.8\%). Cigarette smoking and alcohol consumption 
was present in $19(10.3 \%)$ and $29(18.7 \%)$ of study patients, respectively. Hypertension was present in half of the study patients 78 (50.3\%), while history of diabetes mellitus was present in 31 (20\%) study patients. The mean systolic and diastolic blood pressure were $124 \pm 20 \mathrm{mmHg}$ and $76 \pm 13 \mathrm{mmHg}$ respectively. The mean BMI among study patients was $22.6 \pm 6.3 \mathrm{~kg} / \mathrm{m}^{2}$. Majority of the patient studied had reduced LVEF with a mean of $37.3 \pm 10.7 \%$.

Table 1. Socio-demographic and clinical characteristics of the study patients, $N=155$ 


\begin{tabular}{|c|c|}
\hline Variable & n (\%) / mean (SD) \\
\hline $\begin{array}{l}\text { Age group (years), n (\%) } \\
\leq 25\end{array}$ & $8(5.2)$ \\
\hline $26-35$ & $40(25.8)$ \\
\hline $36-45$ & $26(16.8)$ \\
\hline $46-60$ & $45(29.0)$ \\
\hline$>60$ & $36(23.2)$ \\
\hline Mean (SD) age (years) & $48(16)$ \\
\hline Males, n (\%) & $81(52.3)$ \\
\hline Residing in Dar es salaam, n (\%) & $108(69.7)$ \\
\hline Marital status, n (\%) & \\
\hline Single & $30(19.4)$ \\
\hline Married & $112(72.3)$ \\
\hline Divorced & $9(5.8)$ \\
\hline Widowed & $4(2.6)$ \\
\hline Education, n (\%) & \\
\hline No formal education & $14(9.0)$ \\
\hline Primary & $28(18.1)$ \\
\hline Secondary & $3(1.9)$ \\
\hline High school & $39(25.2)$ \\
\hline College & $71(45.8)$ \\
\hline Taking alcohol, n (\%) & $29(18.7)$ \\
\hline Ever smoked cigarettes, n (\%) & $19(10.3)$ \\
\hline Cardiovascular risk factors, $\mathrm{n}(\%)$ & \\
\hline Hypertension & $78(50.3)$ \\
\hline Diabetes & $31(20)$ \\
\hline Rheumatic heart disease & $47(31.6)$ \\
\hline Arrhythmias & $23(15.5)$ \\
\hline Mean (SD) SBP (mmHg) & $124.8(20.5)$ \\
\hline Mean (SD) DBP (mmHg) & $76.1(13.1)$ \\
\hline Mean (SD) BMI $\left(\mathrm{kg} / \mathrm{m}^{2}\right)$ & $22.6(6.3)$ \\
\hline BMI category, n (\%) & \\
\hline Normal & $152(98.1)$ \\
\hline Underweight & $23(14.8)$ \\
\hline Overweight & $97(62.6)$ \\
\hline Obese & $35(22.6)$ \\
\hline NYHA Functional Status & \\
\hline I & $2(1.3)$ \\
\hline II & $12(7.7)$ \\
\hline III & $93(60.0)$ \\
\hline IV & $48(31.0)$ \\
\hline Mean LVEF (\%) & $37.3(10.7)$ \\
\hline
\end{tabular}

$\mathrm{N}$ : Number of patients, SD: Standard deviation, SBP: Systolic blood pressure, DBP: Diastolic blood pressure, BMI: body mass index was calculated using height and weight (BMI= weight in $\mathrm{kg} /$ height in meters2), NYHA: New York Heart Association. 
Figure 1 shows patients in NYHA classes II, III and IV had high median NT-proBNP levels, while those in class I had normal levels of NT-proBNP.

The spearman correlation ranking test, as plotted in a scatter plot (Figure 2), showed a strong negative correlation between NT-proBNP levels and LVEF $(r=-0.7)$, as the LVEF reduced, the levels of NT-proBNP increased, with a $P$ value of $<0.001$, which was statistically significant.

The predicted cut-off point for readmission was 7,899pg/I of NT-proBNP levels, and majority of the readmitted patients met the above cut-off point. It had a PPV and NPV of $26.76 \%$ and $92.86 \%$ respectively. The sensitivity was $76 \%$ and $60 \%$ specificity, with a P value of 0.004 , which was statistically significant and an AUC of 0.681 (figure 3).

Mortality had an estimated predicted cut-off point of 24,500.3pg/I of NT-proBNP levels, and majority of patients above this cut-off point died. PPV and NPV of $47.37 \%$ and $100 \%$ respectively. The sensitivity was $100 \%$ and $92.54 \%$ specificity, with a $P$ value of 0.00 , which was statistically significant and an AUC of 0.958 (figure 4).

\section{Discussion}

In spite of the advances of new drugs and procedures in recent years, HF patients still are at a higher risk of suffering cardiovascular events and hence being readmitted or die. Studies comparing techniques have shown that there is a good equivalence between both BNP and NT-proBNP methods, although NTproBNP values in $\mathrm{pg} / \mathrm{ml}$ are approximately 8-fold higher than those of BNP. Since there are no studies in Tanzania that have been conducted to address the use of NT-proBNP as marker of HF progression and outcome, to bring awareness, this study shows that, there is a clinical improvement of HF patients when the levels of NT-proBNP are low, and there is poor prognosis in terms of being readmitted, long hospital stay or mortality when the levels are high.

The severe symptomatology of patients with NYHA functional class III or IV patients, added to the presence of cardiogenic shock and the need for hospitalization for cardiac compensation, all identify those who will have the worst disease progression and the highest mortality risk. The present results show that NT-proBNP levels at admission has a positive association with NYHA functional status. After 1 month follow up, the levels of NT-proBNP reduced slightly in those in class II, III and IV. Patients who were in Class II, III and IV, some had a significant decrease in the levels of NT-proBNP, but not to the normal range, this could be contributed to a shorter period of follow up. Although some in these classes (II, III, and IV) still had high or even elevated levels of NT-proBNP, reasons being either due to drug adherence, anaemia or infections. The high levels detected at the first measurement indicate that patients are decompensated, and the maintenance of high levels identifies which patients have a very poor prognosis. These results were similar to that reported by Shang-Chiun Lee in United States, who also found Plasma BNP correlated best with NYHA class. At follow-up, only changes of BNP correlated to changes of NYHA class. BNP decreased in subjects whose NYHA class improved whereas BNP remained unchanged in those whose NYHA class was stable. The current studies, therefore, provide new data on the potential role 
for plasma BNP as an objective biomarker that may help confirm and monitor the impact of therapy upon NYHA class during long-term management of HF to complement clinical judgment.

Although echocardiography is considered the gold standard for the detection of LV dysfunction, it may not always reflect an acute condition. In our study NT-proBNP levels correlated well with LVEF. LVEF can either be reduced or preserved, and in this study, it shows there is a negative correlation between NTproBNP levels and LVEF. The majority of the patients in this study had reduced LVEF, and those with reduced LVEF had the high levels of NT-proBNP $(r=-0.65, P<0.00)$. These finding corresponds well with those observed by Belagavi in India, where he found that the NT-proBNP values increased significantly as the functional severity of HF increased $(P<0.001)$. Groenning and colleagues had similar observation in their study, where patients with systolic dysfunction had higher levels of NT-proBNP compared to those without systolic dysfunction.

Patients with high NT-proBNP at admission had poor prognostic outcome, in terms of clinical progression, readmissions and death. Patients with a cutoff point of $18,000 \mathrm{pg} / \mathrm{l}$ had poor clinical outcome. These patients either after discharge reported recurrence of symptoms within the 30-day period of follow up. Those with mild symptoms and NT-proBNP levels less than 7899pg/I were not readmitted but attended clinic and their management was adjusted accordingly. Those who were readmitted, had NTproBNP levels of above 7,899pg/l, main reasons being infections (Chest infection being major reason) and anaemia. Patients who had $24,500.3 p g / I$ of NT-proBNP levels and above, most of them died. $6 \%$ of patients died within the 1 month follow up period, 2 died before being discharged, 4 within 1 month and 5 after 1 month of follow up. Patients with low NT-proBNP levels at admission has shown to have good prognosis. However, even in a population of patients with the most severe form of the disease, not all of them will die; hence, the early identification of those with the worst clinical progression would allow early referrals to special treatments or transplantation and, hopefully, change the bleak outlook of the disease. Similar studies with same results conducted in Asian countries, including Korea, China, and Japan, with high prevalence of HF due to aging of the population and adoption of a Western lifestyle have revealed that BNP at admission is the most powerful prognostic factor of mortality in patients with HF, and that high levels of BNP are significantly associated with poor outcomes'. Of the four outcomes between mortality, readmission, long hospital stays and poor prognosis, the best predicted by NT-pro BNP is mortality.

The originality of the present study lies in that to date there have been no Tanzanian data available regarding the use of NT-proBNP in the follow-up of patients with heart failure. NT-proBNP values seem to be a very strong predictor of subsequent outcomes in patients admitted for HF and should be used for reducing future cardiovascular events. Our study demonstrates that NT-proBNP could be a powerful tool that can be used to predict readmission and mortality in patients with HF. We have also shown that NTproBNP level can also aid in determining long hospital stay. Thus, the clinical value of NT-proBNP can facilitate discussions with patient prognosis, decisions regarding interventions, and continuity of care. 
Findings of this study calls upon the use of NT-proBNP biomarkers for patients' prognostication in tertiary level facilities.

\section{Declarations}

\section{Ethics approval and consent to participate}

All participants provided written consent before entering the study. The study was approved by the local ethics committee of Muhimbili University of Health and Allied Sciences' Ethical Review Board. Permission to do the study was obtained from Jakaya Kikwete Cardiac Institute management.

\section{Consent for publication}

Not applicable.

\section{Competing interests}

The authors declare no competing interests.

\section{Funding}

Self-funded

\section{Authors' contributions}

MMM, PC and KK conceptualized and designed the study, performed data analysis, interpreted the results and wrote the manuscript.

\section{Acknowledgements}

The authors would like to thank all the institutions, health staff and study participants involved in the study and the following for their technical support; Prof. Mohamed Janabi, Thadei Kavishe and Ponsian Peter.

\section{References}

[1] Executive Summary: HFSA 2006 Comprehensive Heart Failure PracticeGuideline.JCardFail2006; 12:10-38.

[2,3] Stewart S, Mclntyre K, Hole DJ, et al. More 'malignant' than cancer? Five-year survival following a first admission for heart failure. Eur J Heart Fail 2001; 3:315-22. 
[4] H. Dokainish, K. Teo, J. Zhu, A. Roy, K.F. Alhabib, A. Elsayed, L. Palileo-Villaneuva, P. Lopez-Jaramillo, K. Karaye, K. Yusoff, A. Orlandini, K. Sliwa, C. Mondo, F. Lanas, D. Prabhakaran, A. Badr, M. Elmaghawry, A. Damasceno, K. Tibazarwa, E. Belley-Cote, K. Balasubramanian, M.H. Yacoub, M.D. Huffman, K. Harkness, A. Grinvalds, R. McKelvie, S. Yusuf, Heart failure in Africa, Asia, the Middle East, and South America: the INTER-CHF study, Int. J. Cardiol. 204 (2016) 133-141 (Elsevier Ireland Ltd).

[5] Joseph Gallagher et al. Heart Failure in Sub-Saharan Africa. Cardiac Failure Review 2018;4(1):21-4.

[6] Damasceno A, Mayosi BM, Sani M, et al. The causes, treatment, and outcome of acute heart failure in 1006 Africans from 9 countries. Arch Intern Med 2012; 172:1386-94.DOI:

10.1001/archinternmed.2012.3310; PMID: 22945249.

[7] Mayosi BM. Contemporary trends in the epidemiology and management of cardiomyopathy and pericarditis in sub-Saharan Africa. Heart 2007; 93:1176-83. DOI: 10.1136/ hrt.2007.127746; PMID: 17890693.

[8] Tsutamoto T, Wada A, Maeda K, et al. Attenuation of compensation of endogenous cardiac natriuretic peptide system in chronic heart failure: prognostic role of plasma brain natriuretic peptide concentration in patients with chronic symptomatic left ventricular dysfunction. Circulation 1997; 96:509-16.

[9] Yamamoto K, Burnett JCJ, Jougasaki M, et al. Superiority of brain natriuretic peptide as a hormonal marker of ventricular systolic and diastolic dysfunction and ventricular hypertrophy. Hypertension 1996; 28:988-94.

[10] Gardner RS, Ozalp F, Murday AJ, et al. N-Terminal pro-brain natriuretic peptide. A new gold standard in predicting mortality in patients with advanced heart failure. Eur Heart J. 2003; 24: 1735-43.

[11] Cheng V, Kazanegra R, Garcia A, Lenert L, Krishnaswamy P, Gardetto N, Clopton P, Maisel A: A rapid bedside test for B-type peptide predicts treatment outcomes in patients admitted for decompensated heart failure: a pilot study. J Am Coll Cardiol 2001, 37:386-391. 
[12] Cohen-Solal, Logeart D, Bidan Huang, Danlin Cai, Nieminen MS, Mebazaa A: Lowered B-type natriuretic peptide in response to levosimendan or dobutamine treatment is associated with improved survival in patients with severely acutely decompensated heart failure. J Am Coll Cardiol 2009, 53:23432348

[13] Gardner RS, Ozalp F, Murday AJ, et al. N-Terminal pro-brain natriuretic peptide. A new gold standard in predicting mortality in patients with advanced heart failure. Eur Heart J. 2003; 24: 1735-43.

[14] Wong PS, Davidsson GK, Timeyin J, et al. Heart failure in patients admittd to hospital: mortality is still high. Eur J Intern Med. 2002; 13: 304-10.

[15] Gardner RS, Ozalp F, Murday AJ, et al. N-Terminal pro-brain natriuretic peptide. A new gold standard in predicting mortality in patients with advanced heart failure. Eur Heart J. 2003; 24: 1735-43.

[16] Shang-Chiun Lee, Tracy L. Stevens, Sharon M. Sandberg, Denise M. Heublein, Susan M. Nelson, Michihisa Jougasaki, Margaret M. Redfield, John C. Burnett, The potential of brain natriuretic peptide as a biomarker for New York Heart Association class during the outpatient treatment of heart failure, Journal of Cardiac Failure, Volume 8, Issue 3, 2002, Pages 149-154.

[17] Belagavi et al. Correlation between NT proBNP and left ventricular ejection fraction in elderly patients presenting to emergency department with dyspnoea. Indian Heart Journal. 2012: 0019-4832

[18] Groenning et al. Diagnostic and prognostic evaluation of left ventricular systolic heart failure by plasma N-terminal pro-brain natriuretic peptide concentrations in a large sample of the general population. Heart 2004; 90:297-303.

[19] Oliveira Jr MT, Canesin MF, Munhoz RT, et al. Principais características clínicas de pacientes que sobrevivem 24 meses ou mais após uma hospitalização devido a descompensação cardíaca. Arq Bras Cardiol. 2005; 84 (2): 161-6 
[20] Lee JH, et al. Epidemiology of heart failure in Korea: present and future. Korean Circ J. 2016;46(5):658-64.

[21] Kang SH, et al. Prognostic value of NT-proBNP in heart failure with preserved versus reduced EF. Heart. 2015;101(23):1881-8.

[22] Krim SR, et al. Racial/ethnic differences in B-type natriuretic peptide levels and their association with care and outcomes among patients hospitalized with heart failure: findings from get with the guidelinesheart failure. JACC Heart Fail. 2013;1(4):345-52.

\section{Figures}

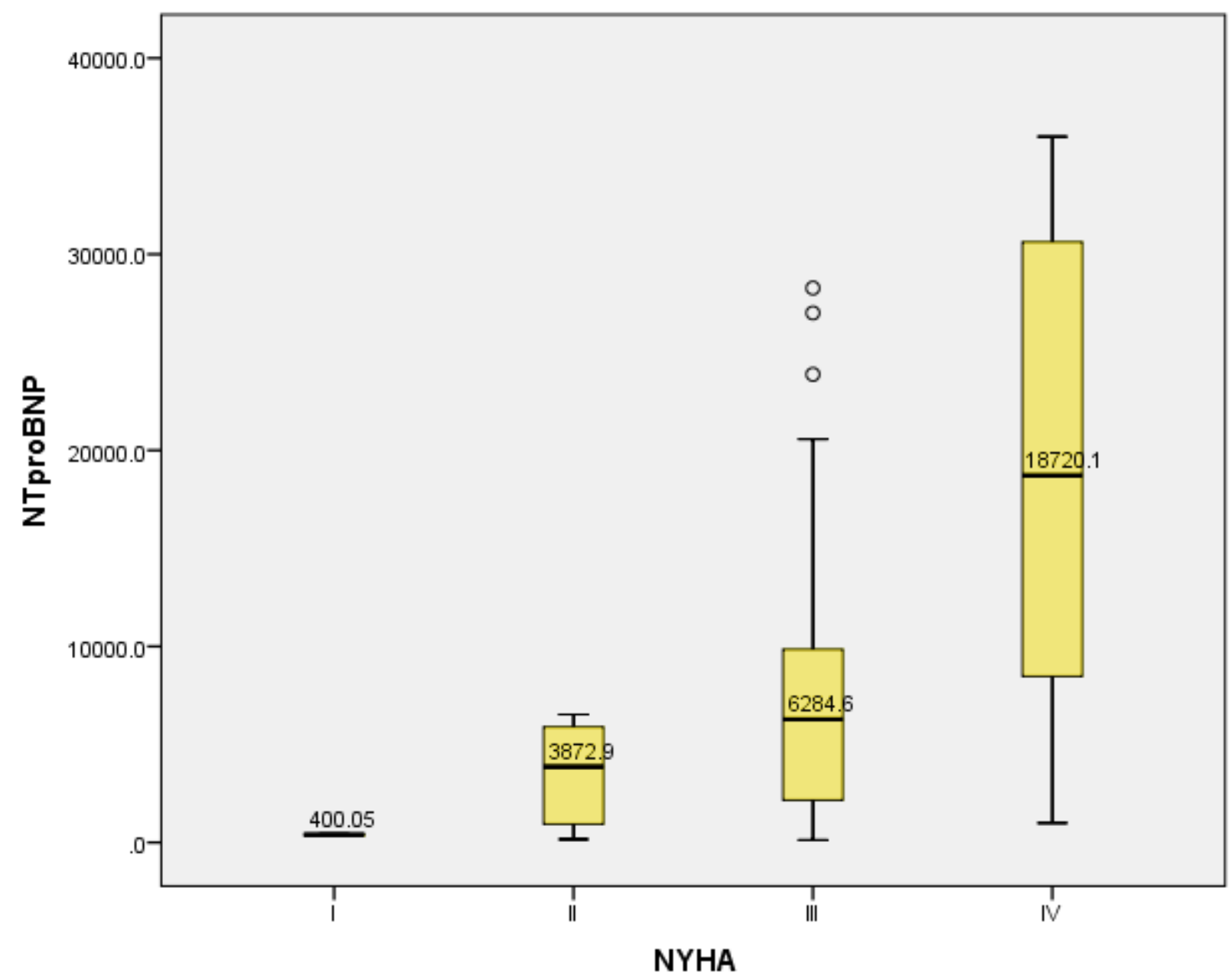

Figure 1 
NT-proBNP levels and NYHA functional status at admission

\section{LVEF vs NT-proBNP}

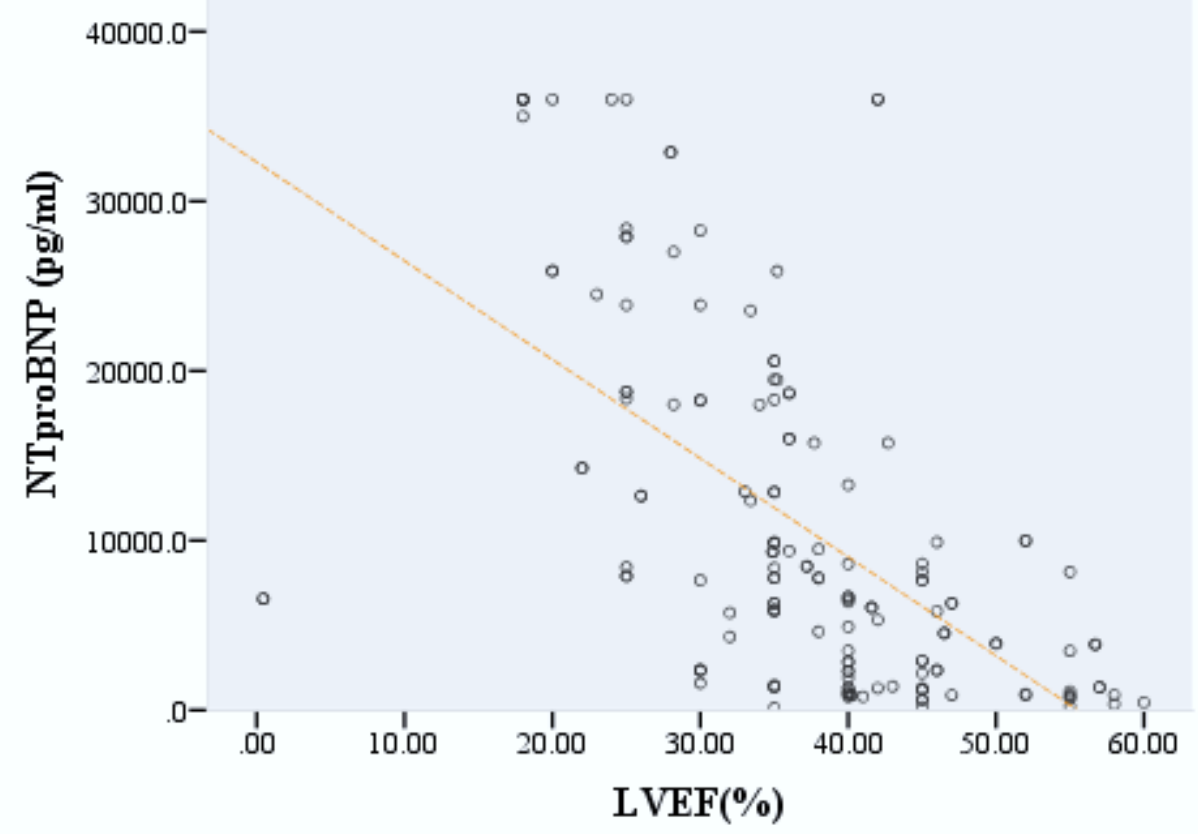

Figure 2

Scatter plot showing correlation between NT-proBNP levels and LVEF at admission

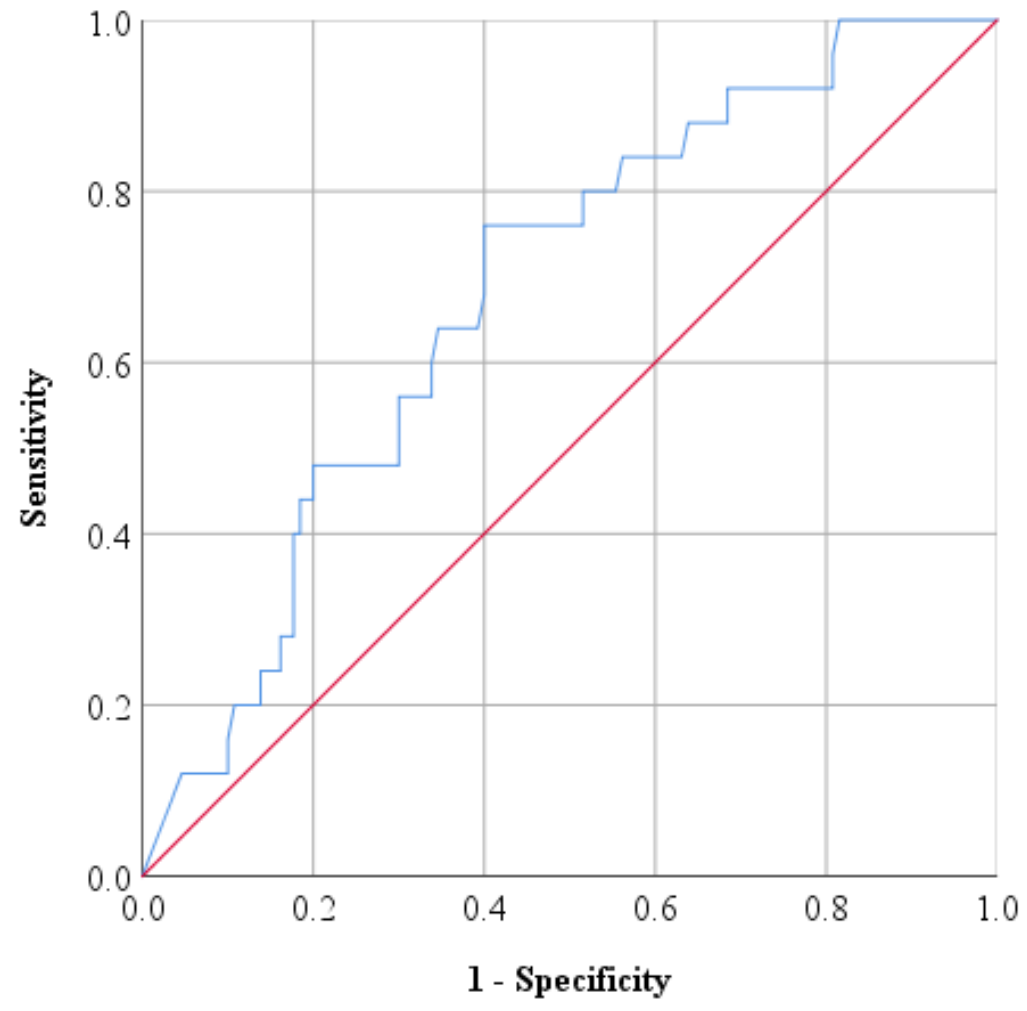

Diagonal segments are produced by ties. 
Figure 3

ROC Curve showing correlation between NT-proBNP levels and readmission

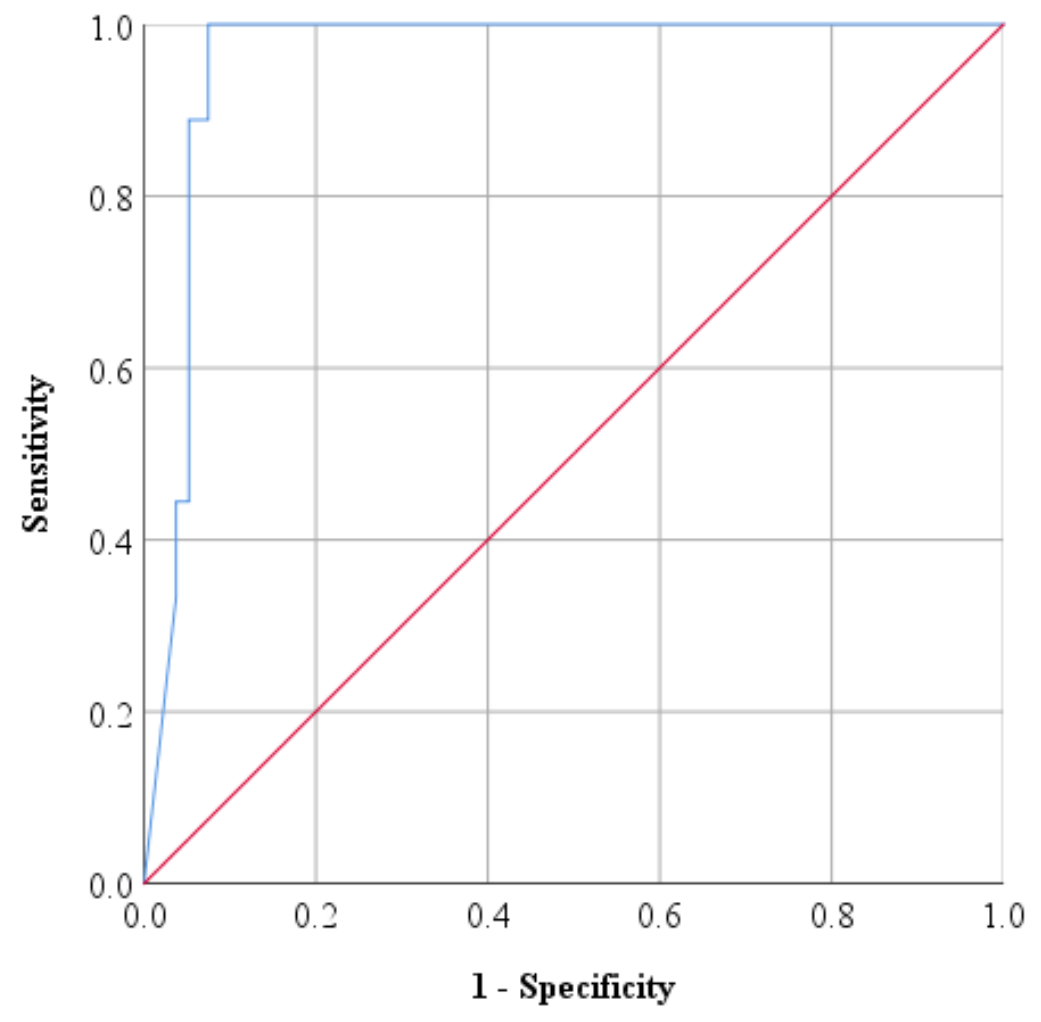

Diagonal segments are produced by ties.

\section{Figure 4}

ROC Curve showing correlation between NT-proBNP and mortality 